\title{
On surfaces with a rectilinear geodesic circle (*)
}

By Anriano Garsia (**) (Poulter Laboratories, Stanford Research Institute, Menlo Park, California and Stanford University)

Summary - We say that a surface has a "rectilinear geodesic circle, if it contains a straight line segment $A B$ and a point $F$ whose geodesic distance from a variable point of $A B$ is a constant.

The problem of generating such surfaces is solved here by constructing families of cones which behave locally (along a curce) like solutions, and then taking their envelopes. This method generates a class of solutions which depend on an arbitrary curve.

\section{INTRODUCTION}

A rectilinear detonation front propagating on a plane sheet of explosive is often used to initiate more elaborated detonation fronts (cfr. [3]). The problem of generating the rectilinear detonation front itself presents some diffi. culties. Such a front is obtained from a detonation that has been started simultaneously along a straight line of a plane sheet of explosive.

Experience has proved that the best way to achieve simultaneity is to use a single detonator, $i$. e., to initiate the detonation at a single point of the explosive. Unfortunately, if a detonation is initiated at some point $F$ of a plane sheet of explosive, the successive detonation fronts will always have the shape of concentric circles with common centers at the point $F$. Therefore the points of a rectilinear target could never be reached simultaneously.

J. O. ERKMaN, of the Poulter Laboratories of Stanford Research Insti. tute, suggested that if the layer of explosive is warped between the initial point $F$ and the rectilinear target $A B$, so as to increase the length of the minimal paths between the point $F$ and the interior points of the segment $A B$, the points of this segment may be reached simultaneously by an explosion started at the point $F$.

If we assume that a detonation on a surface-shaped layer of explosive propagates along geodesics of the surface supporting it, we can conclude that to obtain the desired effect, the triangle $F A B$ should be warped so that it assumes the shape of a surface $S$ in which the segment $A B$ is an arc of a geodesic circle with center in $F$, i.e., so that the geodesic distance in $S$ of any one of the points of $A B$ from the point $F$ is a constant.

$\left(^{*}\right)$ This is an excerpt from a thesis presented in partial fulfillment of the Ph. D. Requiremente, of the Mathematics Department of Stanford University. It was supported in part by the Ballistics Research Laboratory of the Ordnance Department, U. S. Army, under Contract No. DA-01-200-ORD-294.

(**) Now in the Department of Mathematics, Massachusetts Institute of Technology. 
The object of our research has been to investigate the existence of such surfaces in connection with their practical realization.

On a first approach of the problem one can introduce a set of geodesic coordinates $(u, v)$ in the geodesic triangle $F A B$ of our unknown surfaces. Under such coordinate system the lines $u=$ const are the geodesic circles with center in $F$. The parameter $u$ is also chosen so that the segment $A B$ is given by $u=0$, and the point $F$ by $u=l$. The parameter $v$ could be taken to be the ordinary arc tength on $A B$. The lines $v=$ const are the geodesics emanating from the point $F$.

The first fundamental form in these coordinates assumes the familiar expression

$$
d s^{2}=d u^{2}+g^{2}(u, v) d v^{2} .
$$

For such a surface the function $g(u, v)$ cannot be arbitrary, but it must satisfy the conditions

$$
\begin{aligned}
& g(l, v)=0 \\
& g(0, v)=1
\end{aligned}
$$

and

$$
g_{u}(0, v)=0 \text {. }
$$

The first of these conditions is obvious since $u=l$ reduces to the point $F$, and the second is a consequence of the choice of $v$. The third condition is slightly deeper and derives from the fact that $u=0$ must be a geodesie also on the surface.

Introducing the functions $k(u, v), \tau(u, v)$ and $k^{\prime}(u, v)$ to represent the curvature and torsion of the geodesics $v=$ const and the normal currature of the geodesic circles $u=$ const, the second fundamental form writes

$$
I I=k d u^{2}-2 g \tau d u d v+g^{2} h^{\prime} d v^{2} .
$$

It is well known, (see for instance [1]), that the imbedding of the metric (1) can be achieved by integrating the system composed of Gauss " Theorema Egregium :

$$
k k^{\prime}-\tau^{2}=-\frac{g_{u t}}{g}
$$

and the Gauss-Codazzi equations

$$
\begin{gathered}
k_{v}+g \tau_{u}=-2 g_{u} \tau \\
\tau_{v}+g k_{u}^{\prime}=g_{u}\left(k-k^{\prime}\right) .
\end{gathered}
$$

Therefore given a function $g(u, v)$ defined in some rectangle $R\left(0 v^{\prime} ; l, v^{\prime \prime}\right)$ of the plane $(u, v)$ and satisfying $(2),(3)$ and (4), our problem amounts to 
integrating the system (6)-17) throughout some rectangle $R\left(0, v_{A} ; l, v_{B}\right)$ contained in $R\left(0, v^{\prime} ; l, v^{\prime \prime}\right)$ with the side condition

$$
k^{\prime}(0, v)=0 \text {. }
$$

The latter condition together with (4) assures that the line $u=0$ goes onto a rectilinear segment.

Unfortunately it is not difficult to see that the conditions (2), (3) and (4) are by no means sufficient to guarantee that such an imbedding, once started along $A B$ (that is $u=0$ ), can be carried out in the large: that is, to include the point $F(u=l)$.

As a matter of fact one must be very careful with the choice of the function $g(u, v)$ even in order to guarantee the possibility of a real imbedding in the neighbourhood of $A B$. This is because the condition that the line $u=0$ should be straight is very strong.

As an illustration of these difficulties it is worth while to mention that the following inequality

$$
2 u+\int_{v_{1}}^{v_{z}} g(u, v) d v \geq v_{2}-v_{1}
$$

should hold for all $0 \leq u \leq l$ and $v_{A} \leq v_{1}<v_{2} \leq v_{B}$, if we hope to perform our imbedding throughout the rectangle $R\left(0, v_{A} ; l, v_{B}\right)$.

The inequality (9) can be easily deduced as a consequence of the triangular inequality and the fact that $u=0$ is to be a straight line. It seems unlikely that $(9)$ is sufficient to guarantee the imbedding. Actually it is not clear whether this condition is the only one that can be deduced from the geometry of the problem.

An unsolved problem in this direction is the possibility of obtaining one of these surfaces as a bending of surface of revolution. This problem corresponds to assigning $g(u, v)$ satisfying $(2),(3)$ and $(4)$ but depending only on the parameter $u$. Such a surface would be very convenient for the applications since it would be much easier to realize as a piece of hardware $\left({ }^{2}\right)$.

Before concluding this description of the problem. we would like to point out a few interesting facts. Since the geodesics emanating from $F$ on our surfaces meet at right angles the segment $A B$, the integral curvature on the geodesic triangle $A B F$ must be positive. Therefore, on some regions of the triangle $A B F$, the Gadssian curvature of our surfaces must assume positive

(1) Some results have been obtained concerning the possibility of bending a sphere along the equator, but none of these results assure that such a bending can be performed on a strip that would extend up to one of the poles. However this is required in our applications. 
values. This fact implies that in each rectangle $R\left(0, v_{A} ; l, v_{B}\right)$ there are regions where the system $(6)-(7)$ is of elliptic type.

Of course if the functions $k(u, v), \tau(u, v)$ and $k^{\prime}(u, v)$ solutions of $(6)-(7)$ are expected to be real and finite then the GaUssian curvature along $u=0$ cannot be allowed to take positive values This restriction would make the system (6)-(7) parabolic or hyperbolic, (or even mixed, parabolic - hyperbolic), along $u=0$.

The condition (8) makes $u=0$ a characteristic of the system.

\section{A class of solutions.}

\section{A method of solution.}

Our preliminary considerations suggest that the solution of our problem has a certain degree of arbitrariness. This fact is confirmed by the results that we have obtained.

We shall first give a brief description of the method followed.

Let the point $F$ be chosen in $3-$ space and let $d$ be a straight line not containing $F$. We shall assume as a unit of measure the distance of the point $F$ from the straight line $d$.

Let $\{\Gamma(\Psi)\}$ be a one-parameter family of surfaces which has a real envelope $S$ and possesses the following properties:

1) Each surface $\Gamma(\Psi)$ contains both the point $F$ and the straight line $d$.

2) Each surface $\left.\Gamma_{(} \Psi\right)$ touches the envelope $S$ along one of its geodesics emanating from the point $F$. We shall call, this geodesic of contact $\Lambda(\Psi)$, and suppose that it intersects the straight line $d$ at the point $Q(\Psi)$.

3) In each surface $\Gamma(\Psi)$ the arc $Q \bar{F}$ of $\Lambda(\Psi)$ also gives the shortest path between the point $F$ and the straight line $d$. Furthermore, the length of the arc $\widehat{Q F}$ is equal to the same number $l>1$.

The envelope $S$ of such a family is in general a solution of the problem. In fact, $S$ will contain both the point $F$ and the straight line $d$, in addition $S$ will be described by the curves $\Lambda(\Psi)$ that will necessarily have to be geodesics in $S$. The locus of the point $Q(\Psi)$ will then be an arc. of a geodesic circle.

The problem is thus reduced to the construction of such families. It is clear that the method leaves much freedom in the choice of the enveloping surfaces $\Gamma(\Psi)$. As a matter of fact it is easy to see that every solution of our problem can be generated by this method, even if we restrict the surfaces $\Gamma(\Psi)$ to be ruled surfaces.

Actually every solution $S$ whose Gaussian curvature vanishes along the line $d$, and is there sufficiently many times differentiable, can be proved to 
ive the envelope of developable surfaces; in the fashion just described. In this case the surfaces $\Gamma(\Psi)$ are the rectifying developables of the greodesics omanating from the center $F$.

The construction will be carried out here under the restriction that the enveloping surfaces $\Gamma(\Psi)$ should be cones.

We made this choice, of course, because cones are easily described.

\section{Characterization of a collection of cones.}

Let us choose the straight line $d$ to be the $z$-axis of a coordinate system $(x, y, z)$, and the point $F$ to be the point $(0,1,0)$ of the $y$-axis.

Given a real number $l>1$ and a point $V=(0,0,-\gamma)$ of the $z$-axis, we shall say that the cone $\Gamma$ belongs to the collection $O(l, V)$ if it satisfies the following requirements :

1) $\Gamma$ has the vertex at $V$.

2) [' contains both the z-axis and the straight line VF as generators.

3) The shortest path in $\Gamma$ between the point $F$ and the $z$-axis has length equal to $l$.

A cone is completely characterized by its intersection with a sphere having the center at its vertex. Let $\Gamma$ belong to $C(l, V)$ and let $\Sigma_{V}$ be a sphere of unit radius with the center at the point $V$. Let $\Omega$ be the curve intersection of $\Gamma$ and $\Sigma_{V}, \Lambda$ the shortest path in $\Gamma$ between the point $F$ and the $z$-axis, and $Q$ the point where $\Lambda$ meets the $z$-axis.

Finally, let $F^{*}$ and $Q^{*}$ be the points where the generators $V F$ and $V Q$ meet the sphere $\Sigma_{V}$ and $\Psi$ the length of the arc $Q^{*} F^{*}$ of the curce $\Omega$.

If we unfold over a plane the portion of $\Gamma$ limited by $A$, the segment $V Q$, and the segment $V F$, the segments $V Q$ and $V F$ of course will remain rectilinear. Because of the requirements on $\Gamma$, the curve $\Lambda$ will have to go over to the straight line $Q F$ orthogonal to $V Q$, and the length of the segment $Q F$ will have to be equal to $l$. Finally, the curve $\Omega$ will go into the unit circle with the center at $V$. The angle $Q^{*} V F^{*}$ will be equal to $\Psi$.

Thus we can write the following relations:

$$
\begin{gathered}
\overline{V F}=\left|\frac{l}{\sin \Psi}\right| \\
\overline{V Q}=\left|\frac{l}{\tan \Psi}\right| \\
\gamma=\frac{V i^{2}-\sin ^{2} \Psi}{\sin \Psi} .
\end{gathered}
$$


These equations determine the quantities $\overline{V F}, \overline{V Q}$ and $\Psi$ independently of the shape of the cone $\mathrm{I}$.

For a given $l>1$ and $V$ all the cones of $C(l, V)$ unfold over the same triangle $Q V F$ just deseribed. The only arbitrary element in those cones is the shape of the curve $\Omega$, since the length $\Psi$ of the arc $Q^{*} F^{*}$ of $\Omega$ is also determined. Thus any one of those cones can be obtained by prescribing in the sphere $\Sigma_{V}$ an arbitrary eurve $Q$ of length $\Psi=\arcsin \left[l /\left(\sqrt{\left.1+\gamma^{2}\right)}\right)\right]$ joining the two points $Q^{*} F^{*}$ and then projecting $\mathcal{Q}$ from the point $V$.

\section{The parametric equations of the cones of $C(l, V)$.}

Let us assume the point $Q^{*}$ to be the north pole of the sphere $\mathrm{S}_{V}$ and the plane $(x, z)$ the principal meridian plane.

We introduce as parameters in $\Sigma_{V}$ the colatitude $t$ and the longitude $\Phi$.

To describe the curve $Q \mathbf{Q}$ we may introduce the function $t(\Phi)$ giving the colatitude of the point $P^{*}(\Phi)$ of $\boldsymbol{Q}$ in function of its longitude, and the fun. etion $s(\Phi)$ giving the length of the arc $\overline{Q^{*} P^{*}}(\Phi)$.

If we let $\Phi_{0}$ be the limiting longitnde of $\Omega$ at the point $Q^{*}$; then the curve $\mathbf{Q}$ will define a cone of $C(l, V)$ if

$$
\begin{aligned}
& s\left(\Phi_{0}\right)=0 \\
& t\left(\Phi_{0}\right)=0
\end{aligned}
$$

and

$$
\begin{aligned}
& s\left(\frac{\pi}{2}\right)=\Psi \\
& t\left(\frac{\pi}{2}\right)=\arcsin \left\{\frac{\sin \Psi}{l}\right\} .
\end{aligned}
$$

Of course the functions $s(\Phi)$ and $t(\Phi)$ are not independent of each other, they are related by the differential equation

$$
\left(\frac{d s}{d \Phi}\right)^{2}=\left(\frac{d t}{d \Phi}\right)^{2}+\sin ^{2} t
$$

We shall suppose the functions sid and at defined and sufficiently many times differentiable at all points of the interval $\left(\Phi_{0}, \frac{\pi}{2}\right)$.

If $a b c$ are the unit vectors with the directions of the coordinate axis. the position of the point $Q^{*}(\Phi)$ on the curve $Q$ can be described by the vector

$$
k=\sin t \cos \Phi a+\sin t \sin \Phi b+\cos t c .
$$

If $\lambda$ is a variable, the equation of the corresponding cone $\Gamma$ of $C(l, V)$ can be written in the form

$$
\boldsymbol{P}(\lambda, \Phi)=-\gamma c+\lambda \boldsymbol{k} .
$$


The geodesic $\Lambda$ giving the shortest distance, in the cone $\mathrm{r}$, between the point $F$ and the $z$-axis has the equation

$$
\lambda(\Phi)=\frac{l}{\cos s(\Phi)} \frac{1}{\tan \Phi} .
$$

Projecting equation (15) along the coordinate axis and making use of (16) we obtain, for the geodesic $\Lambda$, the following parametric equations

$$
\begin{aligned}
& x(\Phi)=\frac{l}{\tan \Psi} \frac{\sin t}{\cos s} \cos \Phi \\
& y(\Phi)=\frac{l}{\tan \Psi} \frac{\sin t}{\cos s} \sin \Phi \\
& z(\Phi)=\frac{l}{\tan \Psi} \frac{\cos t}{\cos s}-\frac{\sqrt{l^{2}-\sin ^{2} \Psi}}{\sin \Psi} .
\end{aligned}
$$

\section{Construction of families $\{\Gamma(\Psi)\}$.}

If we fix a number $l>1$ and assign for each point $V(0,0,-\gamma)$, or for each value of $\Psi$, a cone $\Gamma$ of $C(l, V)$, we obtain a one-parameter family of cones satislying properties 1) and 3 ) of section 1 . We shall next show in what way the cones of $C(l, V)$ have to be chosen so that property 2$)$ will also be satisfied.

Let us suppose then that we have two functions $s(\Phi, \Psi)$ and $t(\Phi$, 'T) defined in a closed domain $D$ of the $(\tilde{\Phi}$. T) plane of the type

$$
D=\left[(\Phi, \Psi):-\frac{\pi}{2} \leq \Psi \leq \frac{\pi}{2}, \Phi_{0}(\Psi) \leq \Phi \leq \frac{\pi}{2}\right]
$$

satisfying in $D$ the partial differential equation

$$
\left(\frac{\partial s}{\partial \Phi}\right)^{2}=\left(\frac{\partial t}{\partial \Phi}\right)^{2}+\sin ^{2} t
$$

and the boundary conditions

1) along the line $\Phi=\Phi_{0}(\Psi), \quad-\frac{\pi}{2} \leq \psi \leq \frac{\pi}{2}$

$$
\begin{aligned}
& s\left(\Phi_{0}, \Psi\right)=0 \\
& t\left(\Phi_{0}, \Psi\right)=0
\end{aligned}
$$

2) along the line $\Phi=\frac{\pi}{2}, \quad-\frac{\pi}{2} \leq \Psi \leq{ }_{2}^{\pi}$

$$
\begin{aligned}
& s\left(\begin{array}{l}
\pi \\
2
\end{array}, \Psi\right)=\Psi \\
& t\left(\frac{\pi}{2}, \Psi\right)=\arcsin \left\{\frac{\sin \Psi}{l}\right\} .
\end{aligned}
$$


We thus obtain a family of cones whose equations are given by

$$
\boldsymbol{P}(\lambda, \Phi ; \Psi)=-\gamma \boldsymbol{c}+\lambda \boldsymbol{k}(\Phi, \Psi)
$$

where $\gamma=\frac{\sqrt{l^{2}-\sin ^{2} \Psi}}{\sin \Psi}$ and $k$ is given by (14).

In order to find the equation of the envelope of such a family we should intersect each cone with the nearby one; the resulting curves will describe the envelope.

Thus on each cone $\Gamma(\Psi)$ the equation of the curve of contact is given by

$$
\left(\frac{\partial \boldsymbol{P}}{\partial \Psi}, N\right)=0
$$

here $\boldsymbol{N}(\Phi, \Psi)$ represents the normal to the cone $\Gamma(\Psi)$ along the generator $\Phi=$ const.

After a short computation and a few eliminations we obtain from (18) that the curve of contact has the equation

$$
\frac{d \gamma}{d \Psi} \sin t+\lambda \frac{\partial t}{\hat{\imath} \Psi}=0
$$

If we want the curve defined by this equation to coincide with the geodesic $\Lambda(\Psi)$, then equation (19) should be satisfied when we substitute for $\lambda$ the function given by (16).

Performing the substitution and a few eliminations we finally obtain the following partial differential equation in $s(\Phi, \Psi)$ and $t(\Phi, \Psi)$ :

$$
\partial \bar{\partial}=-\frac{l}{\sin \Psi \sqrt{l^{2}-\sin ^{2} \Psi}} \sin t \cos s .
$$

This equation expresses the additional condition that the functions $s$ and $t$ have to satisfy so that the family of cones that they generate will envelope a solution of the problem.

\section{Integration of the system of partial differential equations.}

In view of the results of the previous sections we can conclude that our construction depends on the integration of the system

$$
\begin{gathered}
\left(\frac{\partial s}{\partial \Phi}\right)^{2}=\left(\frac{\partial t}{\partial \Phi}\right)^{2}+\sin ^{2} t \\
\partial t=\frac{l}{\sin \Psi \sqrt{l^{2}-\sin ^{2} \Psi}} \sin t \cos s
\end{gathered}
$$


throughout a closed domain

$$
D=\left[(\Phi, \Psi):-\frac{\pi}{2} \leq \Psi \leq \frac{\pi}{2}, \Phi_{0}(\Psi) \leq \Phi \leq \frac{\pi}{2}\right],
$$

subjected to the boundary conditions

1) along $\Phi=\Phi_{0}(\Psi)$

2) along $\Phi=\frac{\pi}{2}$

$$
\begin{aligned}
& s\left(\Phi_{0}, \Psi\right)=0 \\
& t\left(\Phi_{0}, \Psi\right)=0
\end{aligned}
$$

$$
\begin{aligned}
& s\left(\frac{\pi}{2}, \Psi\right)=\Psi \\
& t\left(\frac{\pi}{2}, \Psi\right)=\arcsin \left\{\frac{\sin \Psi}{l}\right\}
\end{aligned}
$$

Fortunately for our applications the integration of the system (20)-(21) does not offer serious difficulties, in spite of the redundancy of the conditions $(22)-(23)$.

We will not carry out the calculations in detail here, but will instead outline the various steps followed.

If we differentiate equation (20) whith respect to $\Psi$, make repetitive use of (21) and (20) itself, we obtain that the unknown functions $s$ and $t$ should satisfy also

$$
\frac{\partial^{2} s}{\partial \Phi \partial \Psi}=\frac{l}{\sin \Psi \sqrt{l^{2}-\sin ^{2} \Psi}}(\sin s \cos t)_{\Phi}
$$

Performing an integration with respect to $\Phi$ and using the boundary conditions (22)-(23), after short computation and a few cancellations we finally obtain

$$
\frac{\partial s}{\partial \Psi}=\frac{l}{\sin \Psi \sqrt{l^{2}-\sin ^{2} \Psi}} \sin s \cos t
$$

Comparing this new partial differential equation with equation (21) we can easily see that one can be obtained from the other interchanging the roles of $s$ and $t$.

This remarkable fact will allow us to perform our construction by a succession of steps involving only ordinary integration.

It is easy to get from (21) and (24) equations in the sum and the difference of the functions $s$ and $t$. 
The integration of the resulting equations yields

$$
\begin{aligned}
\tan s(\Phi, \Psi) & =\frac{2 m \sigma}{1-m^{2}\left(\sigma^{2}-\rho^{2}\right)} \\
\tan t(\Phi, \Psi) & =\frac{2 m \rho}{1+m^{2}\left(\sigma^{2}-\rho^{2}\right)} .
\end{aligned}
$$

Here $m$ is the function of $\Psi$ defined by

$$
m(\Psi)=\frac{\sin \Psi}{\sqrt{l^{2}-\sin ^{2} \Psi}+l \cos \Psi},
$$

$\sigma$ and $\rho$ are two unknown functions of $\Phi$ defined in the interval $\left(\Phi_{0}, \pi_{\beta 2}\right)$.

The initial conditions (22)-(23), however, can be satisfied if and only if $\sigma$ and $\rho$ satisfy the conditions

and

$$
\begin{aligned}
& \sigma\left(\Phi_{0}\right)=0 \\
& \rho\left(\Phi_{0}\right)=0
\end{aligned}
$$

$$
\begin{aligned}
& \sigma\left(\frac{\pi}{2}\right)=l \\
& \rho\left(\frac{\pi}{2}\right)=1 .
\end{aligned}
$$

This fact is easily seen substituting $s$ and $t$ given by (25)-(26) into (22) and (23). The calculation also yields that the arbitrary function $\Phi_{0}(\Psi)$, describing a portion of the boundary of the domain of integration $D$, should be a constant: a fact that could have been predicted a priori by means of some standard geometrical considerations.

The functions $s$ and $t$ defined by (25)-(26) satisfy equations (21) and (24) but not necessarily (20).

To verify when these functions satisfy also (20) we can either perform a tedious formal substitution of (25)-(26) in (20) or a suitable limit process to evaluate $(20)$ as $\Phi \rightarrow 0$. The latter possibility leads to a much shorter computation. The final result is that $\sigma(\Phi)$ and $\rho(\Phi)$ should be related by the differential equation

$$
\left(\frac{d \sigma}{d \tilde{\Phi}}\right)^{2}=\left(\frac{d \rho}{d \Phi}\right)^{2}+\rho^{2} .
$$

Apart from (28)-(29) and (30) the functions $\sigma$ and $p$ can be arbitrarily prescribed; (25) and (26) will then generate solutions of our problem. 


\section{Geometrical interpretation of the results.}

For every set of values $0 \leq \Phi_{0}<\frac{\pi}{2}, l>1$ and functions $\sigma\left(\Phi_{)},(\rho(\Phi)\right.$ satisfying (28)-(29)-(30) we obtain a solution $S\left(l, \Phi_{0}, \sigma, \rho\right)$ of our geometrical problem.

The surface $S\left(l, \Phi_{\theta}, \sigma, \rho\right)$ assumes a simple shape if $\sigma(\Phi), \rho(\Phi)$ are single-valued non-negative increasing functions of $\Phi$.

The functions $\sigma(\Phi)$ and $\rho(\Phi)$ have an interesting geometrical meaning. In fact, if we let $\Psi \rightarrow 0$ in the equations (17), we obtain

$$
\begin{aligned}
& x(\Phi, 0)=\rho(\Phi) \cos \Phi \\
& y(\Phi, 0)=\rho(\Phi) \sin \Phi \\
& z(\Phi, 0)=0 .
\end{aligned}
$$

This means that $\rho=\rho(\Phi)$ gives the equation in polar coordinates of the section $\Lambda_{0}$ of $S\left(l, \Phi_{n}, \sigma, \rho\right)$ with the plane $(x, y)$. Therefore the conditions $\rho\left(\frac{\pi}{2}\right)=1$ and $\rho\left(\Phi_{0}\right)=0$ express the fact that this curve contains the point $F$ and goes through the origin of the coordinate system with a tangent making an angle equal to $\Phi_{0}$ with the $x$ axis.

Equation $(30)$ then tells us that $\sigma(\Phi)$ is the arc length on $\Lambda_{0}$, while $\sigma\left(\Phi_{0}\right)=0$ and $\sigma\left(\frac{\pi}{2}\right)=l$ mean simply that $\sigma$ is measured from the origin and that the point $F$ has coordinate $l$ in $\Lambda_{0}$.

The last fact is not surprising since $\Psi=0$ is one of the geodesics emanating from $F$.

These results suggest a way to generate functions $\sigma$ and $\rho$ satisfying (28)-(29)-(30) and consequently solutions of the problem.

We simply prescribe in the $(x, y)$ plane a curve $\Lambda_{n}$ joining the point $F$ with the origin of the coordinates. The corresponding solution will be a surface intersecting the $(x, y)$ plane along the curve $\Lambda_{0}$.

To get the most general surfaces of this kind it is however necessary to introduce $\sigma$ as the independent parameter on $\Lambda_{0}$ then get both $\Phi$ and $\rho$ as functions of $\sigma$. With $\sigma$ and $\Psi$ as parameters, equations (17) have meaning even if $\Lambda_{0}$, and thus the corresponding surface $S\left(\Lambda_{n}\right)$, has self intersections.

Let $\mathscr{F}$ denote the class of all these surfaces, i. e., the class of all solutions that are envelopes of a family of cones $\Gamma(\Psi)$.

We shall now describe a few properties common to all surfaces of $F$. Let $S$ be one of them. It is easy to see from our construction, and it can be verified by means of equations (17), that the two geodesics of $S, \Psi=\frac{\pi}{2}$ and $\Psi=-\frac{\pi}{2}$ are rectilinear. Furthermore the points $Q$ and $Q^{\prime}$ of intersection of these two geodesics with the $z$-axis, have coordinates $\left(0,0,-\sqrt{l^{2}-1}\right)$ and 
$\left(0,0,+\sqrt{l^{2}}-1\right)$, respectively. Therefore each surfaces of $\mathcal{F}$ extends in width as much as it possibly ean.

From our construction, it follows also that the surfaces of $F$ are all symmetric with respect to the $(x, y)$ plane and that the curves $\Phi=$ const. are contained, in planes going through the $z$-axis. Thus we can conclude, in virtue of a theorem of Konsres (cfr. [2]) that the curves $\Phi=$ const. on such surfaces are conjugate trajectories to the geodesics $\Psi=$ const.

The latter property oharacterizes the surfaces of $\mathfrak{F}$.

A little manipulation of the parametric equations (17) of the surfaces of $F$ shows also that, independently of the choice of the curve $\Lambda_{0}$ the curves $\Phi=$ const. are conics, generally hyperbolas. These conics have one of their axis of symmetry on the $(x, y)$ plane and each contains the points $Q$ and $Q$ '.

In any solution of our problem the geodesic emanating from $F$ must intersect the rectilinear geodesic circle with an osculating plane orthogonal to it.

This is indeed the case for the curves $\Psi=$ const. of any surfaces of $\mathscr{F}$.

The orthogonality of these osculating planes, together with the constancy of the angle $\Phi_{0}$, allow us to conclude that for a surface of $F$ the tangent plane does not vary along the $z$-axis.

We wish to point out that assigning the curve $\Lambda_{0}$ amounts to prescribing the cylinder $\Gamma(0)$. It is then natural to ask whether any other of the cones $\Gamma(\Psi)$ may also be preseribed arbitrarily.

The answer is affirmative, each of these cones can be assumed to be the initial one to generate a surface of $\mathfrak{F}$.

As long as $\Lambda_{0}$ is a regular curve the points $F, Q, Q^{\prime}$ are in general the only singular points of the corresponding surface $S\left(\Lambda_{0}\right)$ of $\mathfrak{F}$.

In case $\Lambda_{0}$ has singularities, these will propagate along the curves $\Phi=$ const. thronghout $S\left(\Lambda_{0}\right)$.

Two questions arise in connection with the discovery of this class of solutions. The first one is whether or not all these surfaces are essentially different from each other; to be specific whether or not in $F$ there are any couples of isometric surfaces. The second question arises from one of our previons remarks: Can the ourve $\Lambda_{0}$ be chosen in such a way that the corresponding surface $S\left(\Lambda_{0}\right)$ of $\mathscr{F}$ is isometric to a surface of revolution?

We were able to answer both questions after a close study of the first fundamental form of those surfaces. For more details on the calculations the reader may refer to [4] or [5], here we shall state only the results.

The first question has a negative answer, there are no couples of distinct surfaces of $F$ that are inometric. Different curves $\Lambda_{0}$, that is, lead to non isometric surfaces of $\mathfrak{F}$.

The second question has a positive answer, but in way that is not satisfactory from the point of view of the applications. 
The only surface of $\mathfrak{F}$ that is isometric to a surface of revolution is an isometric imbedding of a sector of a plane circle of radius $l$, such that a suitable arc of the circumference is deformed into the segment $\widehat{Q Q^{\prime}}$ of the $z$-axis, the center of the circle becoming the point $F$.

Consequently this imbedding fails to be real in a neighborhood of the rectilinear geodesic circle.

\section{Conclusion.}

The method presented has generated a family of solutions depending on an arbitrary curve; however, these surfaces by no means exhaust the class of all solutions. The same method could be used to treat the general case, or at least the case when the enveloping surfaces are assumed to be developables. But then difficulties arise in handling developables of unknown shapes. Certainly other techniques have to be used.

We are in possession, see [4] or [5], of other classes of solutions some presenting corner-type singularities of the normal, but we have only one example of a solution whose tangent plane varies along the rectilinear geodesic circle, all the surfaces presented here have a constant tangent plane along the $z$-axis.

Some of these surfaces along the rectilinear geodesic circle may have a negative or even a positive Gadsian curvature, but in the regular ones the GaUssian curvature there vanishes.

\section{REFERENCES}

[1] L. Bianuh, Lezioni di Geometria Differenziale, Bologna Nicola Zanichelli, Vol. 1, Part I (1927).

12] G. DakBorx, Lerons sur la Théorie Générale des Surfaces, Paris Ganthior Villars, Livie II, Chap. I (1887) p. 111.

[3] J. O. ERkman and A. Garsia, Simultaneous Detonation of High Explosive Along a Line, - Bulletin of the Ameriean Physical Society , Ser. II Vol. 2 (1957), p. 264.

[1] A. Garsia, On Surfaces with a Rectitinear Geodesic Cucle, Poniter Laboratories, Stan. ford Research Institute Lab. Tech. Report No. 008-57.

[i] A. Gaksia, On Surfaces with a Rectilinear Geodesic Circle, Mic. 58-4351, University Microfilms; 313 No. First Strect, Ann Arbor Michigan, U.S.A. 